\title{
AFRICAN MAHOGANY SUBMITTED TO DRIP IRRIGATION AND FERTILIZATION ${ }^{1}$
}

José Alves Júnior $^{2 *}$, Luis Henrique Antunes Barbosa ${ }^{3}$, Flavio de Oliveira Rosa $^{3}$, Derblai Casaroli ${ }^{4}$, Adão Wagner Pêgo Evangelista ${ }^{4}$ and Lucas Melo Vellame ${ }^{5}$

\footnotetext{
${ }^{1}$ Received on 22.01.2015 accepted for publication on 11.11.2016.

${ }^{2}$ Universidade Federal de Goiás, Escola de Agronomia, Goiânia, GO - Brasil. E-mail: < jose.junior@pesquisador.cnpq.br>.

${ }^{3}$ Universidade Federal de Goiás, mestre em Agronomia, Goiânia, Goiás - Brasil. E-mail: <luishantunes@hotmail.com>and $<$ flaviodeoliveirarosa@gmail.com>.

${ }^{4}$ Universidade Federal de Goiás, Escola de Agronomia, Goiânia, GO - Brasil. E-mail<awpego@pesquisador.cnpq.br>.

${ }^{5}$ Universidade Federal do Recôncavo da Bahia, Centro de Ciências Agrárias, Ambientais e Biológicas, Cruz das Almas, BA - Brasil. E-mail: <lucasvellame@gmail.com>.

*Corresponding author.
}

\begin{abstract}
African mahogany (Khaya ivorensis A. Chev.) is a tree species that has been increasing in Brazilian commercial planting. However, the lack of water and nutrition are great obstacles for crop production. The aim of this study was to evaluate the growth of young mahogany plants submitted to drip irrigation and topdressing. The experimental design was of randomized blocks, with three repetitions in subdivided plots. Treatments consisted of drippers: 1,2 and $3 \mathrm{plt}^{-1}$; flows: 2,4 and $8 \mathrm{~L} \mathrm{~h}^{-1}$, and a treatment without irrigation. For topdressing, subplots levels were, as follows: 1) 17.5 and 25.2; 2) 35.1 and 50.1;3) 52.5 and 75.0; 4) 70.0 and 100.2; and 5) 87.5 and $125.1 \mathrm{~g} \mathrm{plant}^{-1} \mathrm{~N}$ and $\mathrm{K}_{2} \mathrm{O}$, respectively, divided into five bimonthly applications, which started in the $4^{\text {th }}$ month after planting. Plant height, diameter at root collar and at breast height (DBH), and stem height were evaluated. Results showed statistically significant differences $(\mathrm{P}>0.05)$ between irrigated and non-irrigated plants. Mean plant height ranged (from 2 to 20 months in field) from 0.33 to 3.25 and $2.67 \mathrm{~m}$ for irrigated and non-irrigated plants, respectively. Mean stem height ranged from $0.23 \mathrm{~m}$ to 0.87 and $0.71 \mathrm{~m}$ for irrigated and non-irrigated plants, respectively. Thus, irrigation with 1 dripper per tree and flow of $2 \mathrm{~L} \mathrm{~h}^{-1}$ was able to supply mahogany water requirements in the first two years in field. Trees have not responded to $\mathrm{N}$ and $\mathrm{K}$ topdressing at the beginning of the cycle.
\end{abstract}

Keywords: Water stress; Hardwoods; Nitrogen.

\section{MOGNO AFRICANO SUBMETIDO À IRRIGAÇÃO POR GOTEJAMENTO E $A D U B A C ̧ \tilde{A} O$}

\begin{abstract}
RESUMO - O mogno africano (Khaya ivorensis A. Chev.) é uma das espécies arbóreas que vem se destacando no Brasil em plantios comercias. Os déficits hídrico e nutricional são grandes entraves para a produção vegetal. O objetivo deste estudo foi avaliar o crescimento de plantas jovens de mogno submetidas à irrigação por gotejamento e adubação de cobertura. O delineamento experimental foi blocos casualizados - DBC, com três repetições em parcelas subdivididas. Os tratamentos foram: gotejadores: 1, 2 e 3plt ${ }^{-1}$; vazões: 2, 4 e $8 \mathrm{Lh}^{-1}$; e sem irrigação; e as subparcelas, adubação de cobertura: 1) 17,5 e 25,2; 2) 35, 1 e 50,1; 3) 52,5 e 75,0; 4) 70,0 e 100,2; 5) 87,5 e 125, $1 \mathrm{~g}_{\text {planta }^{-1}}$ de $\mathrm{Ne} \mathrm{K} 2 \mathrm{O}$, respectivamente, parceladas em cinco aplicações bimestrais iniciadas no $4^{\circ}$ mês após o plantio. Avaliaram-se: altura de planta, diâmetro de caule no colo e na altura do peito (DAP), e altura de fuste. Os resultados mostraram que houve diferença estatística significativa $(P>0,05)$ entre as plantas irrigadas e não irrigadas. A altura média das plantas variaram (dos 2 aos 20 meses de idade) de 0,33m a 3,25 e 2,67m irrigadas e não irrigadas, respectivamente. Variaram de 0,23
\end{abstract}


m a 0,87 e 0,71 m em altura de fuste, com e sem irrigação. Assim, a irrigação com um gotejador por planta, de vazão $2 L h^{-1}$ foi suficiente para atender as demandas de água do mogno nos primeiros dois anos de cultivo. As plantas não responderam a adubação de $N$ e $K$.

Palavras-chave: Déficit hídrico; Madeira nobre; Nitrogênio.

\section{NTRODUCTION}

The use of forest products currently faces the problem of raw material decrease in the sector, both due to ecological pressures, aimed at reducing exploitation of native forests, as well as due to the scarcity of forest products, which are increasingly distant from consumer areas. Among the affected sectors, sawmills and laminations are highlighted, which, in Brazil, survive from native forest extraction regarding "hardwood".

In order for planted forests to serve the consumer market, there is the need to choose the appropriate species and silvicultural techniques to be employed. In addition, forests must produce timber in quality and quantity compatible with market expectations. Among hardwood exotic species introduced in Brazil, the African mahogany (Khaya ivorensis A. Chev.) stands out. The species aforementioned had its origin in countries from the west coast of the African continent, which has similar edaphoclimatic characteristics to some Brazilian regions, explaining the rapid adaptation of the species in Brazil. For Brito et al. (2013), a large part of the territory of Goiás State has edaphoclimatic aptitude for African mahogany cultivation, which may represent the addition of one more crop with good economic profitability for the State and perhaps for other places with similar characteristics.

Among factors limiting plant production, water deficit is highlighted, which occurs in large cultivable areas, affecting plant-water relations and plant metabolism (Nogueira et al., 2000). Additional water supply through irrigation allows species cultivation outside their natural environments, ensuring good growth and plant development, which is observed in their yield. Studies have demonstrated the positive effect of irrigation on arboreal species growth and yield for guava (Silva, 2012), citrus (Alves Júnior et al., 2011), and eucalyptus (Lopes et al., 2007). On the other hand, some plants do not respond to irrigation or fertilization, as is the case of pequi (Alves Junior et al., 2013). In addition, tree species have nutrient demand variation depending on the species, development stage and climatic conditions (Fernandes et al., 2000).

Due to the limited water resources in many regions, localized drip irrigation is a great alternative, because it has high water application efficiency.

Many studies have argued that water and nutritional availability are the most limiting factors for plant development in the Cerrado biome. Thus, the objective of this study was to evaluate the growth of African mahogany plants submitted to irrigation and fertilization, considering the potential for exploitation of African mahogany and the lack of information on this forest species in Brazil.

\section{MATERIALAND METHODS}

The experiment was conducted in an experimental area in Bonfinópolis, GO (16³5'49" S; 49¹6’39" W, elevation of $780 \mathrm{~m}$ ), with annual mean temperature of $23{ }^{\circ} \mathrm{C}$, relative humidity of $71 \%$ and accumulated rainfall of $1487 \mathrm{~mm}$. The study location has well-defined dry (May-September) and rainy (October-April) seasons. According to Köppen, climate was defined as Aw, tropical savannah, megathermal. Mean annual evaporation (Class A pan) is $1915 \mathrm{~mm}$. The strongest winds are recorded in September (Silva et al., 2007). In the region, a dystroferric Red Latosol (Oxisol), clay texture, Cerradão subperennial stage and flat relief predominates (Silva et al., 2007). Soil analysis was performed before preparation of the area for two depths, 0-20 and 20-40 cm, and the following chemical characteristics were found: $\mathrm{pH}\left(\mathrm{CaCl}_{2}\right)=5.1$ and 5.0; $\mathrm{OM}=2.1$ and $1.2 \%$; $($ Mehlich $)=4.2$ and $1.4 \mathrm{mg} \mathrm{dm}^{-3}$; $\mathrm{Al}=0.0$ and $0.0 \mathrm{mmol}_{\mathrm{c}} \mathrm{dm}^{-3} ; \mathrm{H}+\mathrm{Al}=2.8$ and $2.8 \mathrm{mmol}_{\mathrm{c}} \mathrm{dm}^{-3}$; $\mathrm{K}=45.0$ and $26.0 \mathrm{mg} \mathrm{dm}^{-3} ; \mathrm{Ca}=0.9$ and $0.5 \mathrm{mmol}_{\mathrm{c}} \mathrm{dm}^{-3}$; $\mathrm{Mg}=0.3$ and $0.2 \mathrm{mmol} \mathrm{dm}^{-3} ; \mathrm{CEC}=4.1$ and $3.6 \mathrm{mmol} \mathrm{dm}^{-3}$; $\mathrm{V}(\%)=32.0$ and $21.5 \%$. Moreover, the following physical characteristics were found: Sand $=38.0$ and $47.0 \%$; Silt $=24.0$ and $23.0 \%$ and Clay $=38.0$ and $30.0 \%$ (clay loam texture) with water retention estimated at 1.5 $\mathrm{mm} \mathrm{cm}^{-1}$. 
The experiment was carried out in the field (March/ 2012), containing 450 African mahogany plants (Khaya ivorensis A. Chev.) with approximately 30 days old (mean diameter: $0.8 \mathrm{~cm}$; mean height: $32 \mathrm{~cm}$; stem height $7.5 \mathrm{~cm}$ ) and $5 \times 5 \mathrm{~m}$ spacing. Area preparation was carried out in August 2011 ( 3 months before planting) with disk plowing and two harrows, in which dolomitic limestone was added, increasing the base saturation to $70 \%$. The irrigation system was installed in April 2012. At planting, holes were fertilized with $100 \mathrm{~g} \mathrm{P}_{2} \mathrm{O}_{5}$ (single superphosphate). Immediately after planting, $5 \mathrm{~L}$ of water was placed per plant, including the nonirrigated treatment, in order to guarantee survival. Rainfall equal to $2216.2 \mathrm{~mm}$ was recorded during the experiment period (March/2012 to November/2013).

A Randomized Complete Block (RCB) experimental design was used, with three repetitions in subdivided plots and 50 treatments. Ten irrigation plots (Table 1) were subdivided into 5 topdressing doses (Table 1). Each experimental plot consisted of 15 plants, with 3 plants for each subplot, totaling 150 plants per block and 450 plants in the experiment. In addition to border plants, the experimental area had 600 plants (totaling an area of 1.5 ha). Irrigation started in May 2012 (dry period) using the drip system, with 1, 2 and 3 drippers per plant and 2, 4 and $8 \mathrm{~L} \mathrm{~h}^{-1}$ (self-compensating) flows, plus the control, without irrigation. Netafim brand drippers, PCJ-CNL model, were used. Plot 6, containing two drippers per plant and a flow rate of $4 \mathrm{~L} \mathrm{~h}^{-1}$, was used to apply the standard water volume, i.e., the volume calculated to restore $100 \%$ of plant water requirements. In addition, four plots ( 2 to 5 ) were irrigated below the standard volume, and four plots ( 7 to 10 plots) were irrigated above the standard. Fertilization began in July 2012, with the conduction of the first topdressing. The fertilization dose used in the control was recommended by silviculturists of the region (subplot 3), $52.5 \mathrm{~g} \mathrm{~N}$ and $75 \mathrm{~g}\left(\mathrm{~K}_{2} \mathrm{O}\right)$ per plant, annually. From the standard dose, smaller and larger doses were applied to the subplots (Table 1). In addition, the total dose value was divided equally and distributed bimonthly, manually and circularly in the subplots, at $0.30 \mathrm{~m}$ from the plant. Plants that received fertilization treatments were marked with colored ribbons, where each color corresponded to a fertilization dose.

Table 1 - Plot descriptions: number of drippers per tree, individual dripper flow $\left(\mathrm{L} \mathrm{h}^{-1}\right)$, wet area per tree $\left(\mathrm{A}, \mathrm{m}^{2}\right)$, water volume applied at 20 months in field $\left(\mathrm{Vol}, \mathrm{m}^{3}\right.$ plant $\left.^{-1}\right)$; and experimental subplots description: ammonium sulfate $\left(\mathrm{NH}_{4} \mathrm{SO}_{4}\right)$ and potassium chloride $(\mathrm{KCl})$ leves used in the five fertilization subplots, bimonthly and annually, for African mahogany trees in Bonfinópolis, GO, Brazil.

Tabela 1 -Descrição das parcelas experimentais: número de gotejadores por planta, Vazão individual do gotejador $\left(L h^{-1}\right)$, Área molhada por planta $\left(A, m^{2}\right)$,Volume de água aplicado no período de 20 meses de avaliação, por planta $\left(V o l, \mathrm{~m}^{3}\right.$ planta-1); e descrição das subparcelas experimentais: Dosagens de sulfato de amônio ( $\mathrm{NH}_{4} \mathrm{SO}_{4}$ ) e cloreto de potássio (KCl) utilizadas nas cinco subparcelas de adubação, bimestral e anual, por planta de Mogno Africano, em Bonfinópolis-GO.

\begin{tabular}{|c|c|c|c|c|}
\hline Plot & No. of drippers Per plant & Flow rate $\left(\mathrm{L} \mathrm{h}^{-1}\right)$ & Wet area $\left(\mathrm{m}^{2}\right)$ & $\operatorname{Vol}\left(\mathrm{m}^{3}\right.$ plant $\left.^{-1}\right)$ \\
\hline 1 & 0 & - & 0.44 & 13.44 \\
\hline 2 & 1 & 2 & 0.29 & 8.96 \\
\hline 3 & 2 & 2 & 0.15 & 4.48 \\
\hline 4 & 3 & 2 & 0.27 & 6.72 \\
\hline 5 & 1 & 4 & 0.18 & 4.48 \\
\hline 6 & 2 & 4 & 0.09 & 2.24 \\
\hline 7 & 3 & 4 & 0.22 & 3.36 \\
\hline 8 & 1 & 8 & 0.10 & 2.24 \\
\hline 9 & 2 & 8 & 0.05 & 1.12 \\
\hline 10 & 3 & 8 & - & - \\
\hline \multirow[t]{3}{*}{ Subplot } & \multicolumn{2}{|c|}{ Bimestral } & \multicolumn{2}{|c|}{ Annual } \\
\hline & $\mathrm{N}$ & $\mathrm{K}_{2} \mathrm{O}$ & $\mathrm{N}$ & $\mathrm{K}_{2} \mathrm{O}$ \\
\hline & \multicolumn{4}{|c|}{ 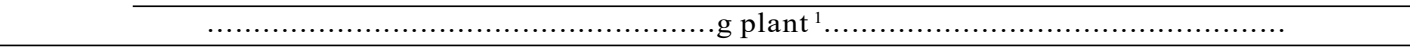 } \\
\hline 1 & 3.5 & 5.0 & 17.5 & 25.2 \\
\hline 2 & 7.0 & 10.0 & 35.1 & 50.1 \\
\hline 3 & 10.5 & 15.0 & 52.5 & 75.0 \\
\hline 4 & 14.0 & 20.0 & 70.0 & 100.2 \\
\hline 5 & 17.5 & 25.0 & 87.5 & 125.1 \\
\hline
\end{tabular}


For the first year, irrigation was performed during the period from May to October 2012, and in the second year, irrigation was carried out from June to September 2013. In irrigated treatments, irrigations were performed daily, and the applied water amount was estimated from crop evapotranspiration (ETc), which is the product of reference evapotranspiration (ETo) and crop coefficient (Kc). ETo was estimated by Penman-Monteith equation and the adopted Kc was 0.5 for the first year and 0.7 for the second. Kc data was obtained from fruit plant studies due to lack of information on African mahogany.

The meteorological variables of the experimental area (minimum and maximum air temperatures, solar radiation, relative air humidity, wind speed and rainfall) were collected with the aid of an automated weather station (Davis Vantage PRO2).

Gross irrigation volume was calculated in relation to the wet area in each treatment. The wet area (WA) was determined from the diameter wetted by the bulb, estimated by the model proposed by Schwartzman; Zur (1986). Irrigation application efficiency was 90\%.

The volume of water applied per plant $\left(\mathrm{Vol}, \mathrm{m}^{3}\right)$ and the irrigation time $(I T, \mathrm{~h})$ were obtained by equations [1] and [2], respectively:

$$
\begin{aligned}
& \text { Vol }=E T o \cdot K C \cdot K l o c \cdot A P \\
& I T=\frac{(q / e f)}{V o l}
\end{aligned}
$$

Where:

$K c$ is the crop coefficient ( 0.5 for the first year and 0.7 for the second), $K_{l o c}$ is the evapotranspiration reduction coefficient for localized irrigation, $A P$ is the useful area of each plant $\left(\mathrm{m}^{2}\right), q$ is the dripper flow rate $\left(\mathrm{L} \mathrm{h}^{-1}\right)$, and $e f$ is the water application efficiency $(90 \%)$. For $K_{l o c}$ calculation with wet area percentage of less than 20\% (Fereres, 1981, apud Mantovani et al., 2009), equation 3 was used:

$$
K_{\mathrm{loc}}=1.94 P M+0.1
$$

Where $P M$ is the wet area percentage, in absolute value.

The wet diameter $(\mathrm{D}, \mathrm{m})$ was obtained from the model proposed by Schwartzman \& Zur (1986):

$$
D=1.82 \cdot \operatorname{Vol}^{0.22}\left(\frac{K s}{q}\right)^{-0,17}
$$

Where $\mathrm{Ks}$ is the saturated soil hydraulic conductivity $\left(\mathrm{m} \mathrm{s}^{-1}\right)$ and $q$ is the dripper flow rate, given in $\mathrm{m}^{3} \mathrm{~s}^{-1}$.

Plant growth assessments were conducted every two months (May, July, September and November 2012, and January, March, May 2013) by measuring the following phenometric variables: plant height - measured from the upper end of the orthotropic branch to ground level, using a millimeter precision ruler; stem height - measured from ground level to the first leaf insertion; and trunk diameter - carried out five centimeters from the ground, with the aid of a pachymeter.

For data statistical analysis, the SISVAR software - Variance Analysis System, was used. For irrigation treatments, analysis was qualitative between treatments and in time, and mean comparison was conducted by Tukey's test at 5\% error probability. For fertilization treatments, data quantitative analysis was made through regression analysis.

\section{RESULTS}

Plant growth in both height and diameter was influenced by irrigation, showing statistical differences (Table 2) between irrigated and non-irrigated treatments ( $>0.05$ ). Height growth rate was $25 \%$ higher in irrigated treatments compared to the non-irrigated treatment. Mean plant height at the beginning of the study was of 0.33 $\mathrm{m}$ (2 months of age). In the last evaluation, plants reached $3.25 \mathrm{~m}$ and $2.67 \mathrm{~m}$ (20 months of age) when irrigated and not irrigated, respectively. Stem height growth rate was $33 \%$ higher, starting with a mean of $0.23 \mathrm{~m}$, at 2 months of age, and reaching $0.87 \mathrm{~m}$ and $0.71 \mathrm{~m}$ at 20 months of age, when irrigated and not irrigated, respectively. Diameter at root collar growth rate was $19 \%$ higher, starting with a mean of $0.79 \mathrm{~cm}$ ( 2 months of age), reaching $5.9 \mathrm{~cm}$ and $5.1 \mathrm{~cm}$ at 16 months of age in irrigated and non-irrigated plants, respectively. The diameter at breast height (DBH) growth rate was $24 \%$ higher, reaching 20 months $4.6 \mathrm{~cm}$ and $3.7 \mathrm{~cm} \mathrm{a} 20$ months of age for irrigated and non-irrigated plants, respectively.

Plant heights in irrigated treatments differed from the non-irrigated treatment from July 2013 (Table 2), the $4^{\text {th }}$ month after planting (full drought season).

It is noteworthy that, even with the beginning of rainfall in October, non-irrigated plants failed to obtain enough growth rates to match irrigated plant means (Table 2). For stem height, this effect was only observed from the beginning of the drought period 
Table 2 - Mean total height, stem height, stem diameter and DBH data evaluations subjected to irrigated and non-irrigated treatments in Bonfinópolis, GO, Brazil.

Tabela 2 - Médias dos dados das avaliações de: altura total, altura de fuste, diâmetro de caule e DAP em metros, submetidas aos tratamentos de irrigação e sem irrigação, Bonfinópolis-Go.

\begin{tabular}{|c|c|c|c|c|c|c|c|c|c|c|c|c|c|c|c|}
\hline & \multicolumn{10}{|c|}{ Plant Height } & \multicolumn{5}{|c|}{ Stem Height } \\
\hline & \multicolumn{4}{|c|}{2012} & \multicolumn{6}{|c|}{2013} & \multicolumn{4}{|c|}{2012} & \multirow{2}{*}{$\frac{2013}{\operatorname{Jan}}$} \\
\hline & May & Jul & Sep & Nov & Jan & Mar & May & Jul & Sep & Nov & May & Jul & Sep & Nov & \\
\hline & 60 & 120 & 180 & 240 & 380 & 440 & 500 & 560 & 620 & 680 & 60 & 120 & 180 & 240 & 380 \\
\hline $8 \mathrm{~L} / \mathrm{h}$ & 0.32 & 0.38 & 0.49 & 0.64 & 0.81 & 1.49 & 2.14 & 2.33 & 2.82 & 3.28 & 0.22 & 0.28 & 0.30 & 0.29 & 0.32 \\
\hline 3 got/pl & $\mathrm{aF}$ & $\mathrm{aF}$ & $\mathrm{aEF}$ & $\mathrm{aEF}$ & $\mathrm{aE}$ & $\mathrm{aD}$ & $\mathrm{aC}$ & $\mathrm{abC}$ & $\mathrm{aB}$ & $\mathrm{aA}$ & $\mathrm{aE}$ & $\mathrm{aE}$ & $\mathrm{aE}$ & $\mathrm{aE}$ & $\mathrm{aE}$ \\
\hline $8 \mathrm{~L} / \mathrm{h}$ & 0.29 & 0.35 & 0.46 & 0.58 & 0.79 & 1.39 & 2.06 & 2.27 & 2.74 & 3.17 & 0.21 & 0.26 & 0.29 & 0.28 & 0.29 \\
\hline 2 got/pl & $\mathrm{aF}$ & $\mathrm{aF}$ & $\mathrm{aEF}$ & $\mathrm{aEF}$ & $\mathrm{aE}$ & $\mathrm{aD}$ & $\mathrm{aC}$ & $\mathrm{abC}$ & $\mathrm{aB}$ & $\mathrm{aA}$ & $\mathrm{aE}$ & $\mathrm{aE}$ & $\mathrm{aE}$ & $\mathrm{aE}$ & $\mathrm{aE}$ \\
\hline $8 \mathrm{~L} / \mathrm{h}$ & 0.33 & 0.38 & 0.47 & 0.63 & 0.82 & 1.52 & 2.13 & 2.38 & 2.78 & 3.25 & 0.24 & 0.26 & 0.29 & 0.27 & 0.28 \\
\hline 1 got/pl & $\mathrm{aF}$ & $\mathrm{aF}$ & $\mathrm{aEF}$ & $\mathrm{aEF}$ & $\mathrm{aE}$ & $\mathrm{aD}$ & $\mathrm{aC}$ & $\mathrm{aBC}$ & $\mathrm{aB}$ & $\mathrm{aA}$ & $\mathrm{aE}$ & $\mathrm{aE}$ & $\mathrm{aE}$ & $\mathrm{aE}$ & $\mathrm{aE}$ \\
\hline $4 \mathrm{~L} / \mathrm{h}$ & 0.33 & 0.38 & 0.50 & 0.66 & 0.85 & 1.50 & 2.04 & 2.23 & 2.61 & 3.15 & 0.22 & 0.25 & 0.29 & 0.28 & 0.32 \\
\hline 3 got/pl & $\mathrm{aF}$ & $\mathrm{aF}$ & $\mathrm{aEF}$ & $\mathrm{aEF}$ & $\mathrm{aE}$ & $\mathrm{aD}$ & $\mathrm{aC}$ & $\mathrm{abBC}$ & $\mathrm{abB}$ & $\mathrm{aA}$ & $\mathrm{aD}$ & $\mathrm{aD}$ & $\mathrm{aD}$ & $\mathrm{aD}$ & $\mathrm{aD}$ \\
\hline $4 \mathrm{~L} / \mathrm{h}$ & 0.32 & 0.37 & 0.47 & 0.61 & 0.79 & 1.44 & 2.09 & 2.27 & 2.77 & 3.27 & 0.22 & 0.26 & 0.27 & 0.27 & 0.30 \\
\hline $2 \mathrm{got} / \mathrm{pl}$ & $\mathrm{aF}$ & $\mathrm{aF}$ & $\mathrm{aEF}$ & $\mathrm{aEF}$ & $\mathrm{aE}$ & $\mathrm{aD}$ & $\mathrm{aC}$ & $\mathrm{abC}$ & $\mathrm{aB}$ & $\mathrm{aA}$ & $\mathrm{aE}$ & $\mathrm{aE}$ & $\mathrm{aE}$ & $\mathrm{aE}$ & $\mathrm{aE}$ \\
\hline $4 \mathrm{~L} / \mathrm{h}$ & 0.31 & 0.35 & 0.47 & 0.60 & 0.79 & 1.43 & 2.01 & 2.27 & 2.74 & 3.26 & 0.22 & 0.24 & 0.27 & 0.27 & 0.28 \\
\hline $1 \mathrm{got} / \mathrm{pl}$ & $\mathrm{aF}$ & $\mathrm{aF}$ & $\mathrm{aEF}$ & $\mathrm{aEF}$ & $\mathrm{aE}$ & $\mathrm{aD}$ & $\mathrm{aC}$ & $\mathrm{abC}$ & $\mathrm{abC}$ & $\mathrm{aA}$ & $\mathrm{aE}$ & $\mathrm{aE}$ & $\mathrm{aE}$ & $\mathrm{aE}$ & $\mathrm{aE}$ \\
\hline $2 \mathrm{~L} / \mathrm{h}$ & 0.33 & 0.38 & 0.49 & 0.65 & 0.80 & 1.42 & 1.93 & 2.25 & 2.71 & 3.15 & 0.22 & 0.24 & 0.28 & 0.28 & 0.30 \\
\hline $3 \operatorname{got} / \mathrm{pl}$ & $\mathrm{aF}$ & $\mathrm{aF}$ & $\mathrm{aEE}$ & $\mathrm{aEF}$ & $\mathrm{aE}$ & $\mathrm{aD}$ & $\mathrm{aC}$ & $\mathrm{abC}$ & $\mathrm{abB}$ & $\mathrm{aA}$ & $\mathrm{aE}$ & $\mathrm{aE}$ & $\mathrm{aE}$ & $\mathrm{aE}$ & $\mathrm{aE}$ \\
\hline $2 \mathrm{~L} / \mathrm{h}$ & 0.35 & 0.41 & 0.50 & 0.62 & 0.80 & 1.50 & 2.18 & 2.47 & 2.95 & 3.43 & 0.24 & 0.27 & 0.30 & 0.30 & 0.31 \\
\hline 2 got/pl & $\mathrm{aF}$ & $\mathrm{aF}$ & $\mathrm{aEF}$ & $\mathrm{aEF}$ & $\mathrm{aE}$ & $\mathrm{aD}$ & $\mathrm{aD}$ & $\mathrm{aC}$ & $\mathrm{aB}$ & $\mathrm{aA}$ & $\mathrm{aE}$ & $\mathrm{aE}$ & $\mathrm{aE}$ & $\mathrm{aE}$ & $\mathrm{aE}$ \\
\hline $2 \mathrm{~L} / \mathrm{h}$ & 0.36 & 0.40 & 0.51 & 0.66 & 0.80 & 1.50 & 2.10 & 2.38 & 2.83 & 3.34 & 0.26 & 0.28 & 0.31 & 0.33 & 0.33 \\
\hline 1got/pl & $\mathrm{aF}$ & $\mathrm{aF}$ & $\mathrm{aEF}$ & $\mathrm{aEF}$ & $\mathrm{aE}$ & $\mathrm{aD}$ & $\mathrm{aC}$ & $\mathrm{aC}$ & $\mathrm{aB}$ & $\mathrm{aA}$ & $\mathrm{aD}$ & $\mathrm{aD}$ & $\mathrm{aD}$ & $\mathrm{aD}$ & $\mathrm{aD}$ \\
\hline Sem & 0.33 & 0.38 & 0.39 & 0.43 & 0.64 & 1.19 & 1.82 & 1.93 & 2.31 & 2.67 & 0.28 & 0.26 & 0.26 & 0.27 & 0.29 \\
\hline \multirow[t]{5}{*}{ Irrig. } & $\mathrm{aF}$ & $\mathrm{aF}$ & $\mathrm{aF}$ & $\mathrm{aE}$ & $\mathrm{aE}$ & $\mathrm{aD}$ & $\mathrm{aC}$ & $\mathrm{bBC}$ & $\mathrm{bAB}$ & $\mathrm{bA}$ & $\mathrm{aE}$ & $\mathrm{aE}$ & $\mathrm{aE}$ & $\mathrm{aE}$ & $\mathrm{aE}$ \\
\hline & \multicolumn{7}{|c|}{ Diameter at root collar } & \multicolumn{3}{|c|}{$\begin{array}{l}\text { Diameter at } \\
\text { breast height }\end{array}$} & \multicolumn{5}{|c|}{ Stem height } \\
\hline & \multicolumn{4}{|c|}{2012} & \multicolumn{6}{|c|}{$201 \overline{3}$} & \multicolumn{5}{|c|}{2013} \\
\hline & May & Jul & Sep & Nov & Jan & Mar & May & Jul & Sep & $100 \mathrm{~V}$ & IVIal & May & Jul & Sep & Nov \\
\hline & 60 & 120 & 0 & 240 & 380 & 440 & 500 & 560 & 620 & 680 & 440 & 500 & 560 & 20 & 680 \\
\hline $8 \mathrm{~L}$ & 0.0078 & 0.01 & 0.015 & 0.019 & 0.026 & 0.045 & 0.058 & 0.037 & 0.043 & 0.048 & 0.51 & 0.64 & 0.68 & 0.76 & 0.87 \\
\hline & & $\mathrm{aB}$ & & & $\mathrm{aDE}$ & $\mathrm{aEF}$ & & & $\mathrm{aAB}$ & & $\mathrm{aD}$ & $\mathrm{aC}$ & $\mathrm{aBC}$ & $\mathrm{aB}$ & $\mathrm{aA}$ \\
\hline $8 \mathrm{~L} / \mathrm{h}$ & 0.0076 & 0.009 & 0.014 & 0.017 & 0.025 & 0.042 & 0.058 & 0.034 & 0.039 & 0.044 & 0.49 & 0.59 & 0.62 & 0.70 & 0.87 \\
\hline $2 \mathrm{got} / \mathrm{pl}$ & $\mathrm{aA}$ & $\mathrm{aB}$ & & $\mathrm{aD}$ & $\mathrm{aDE}$ & $\mathrm{aEF}$ & $\mathrm{aF}$ & $\mathrm{abB}$ & $\mathrm{abAB}$ & $\mathrm{abA}$ & $\mathrm{abD}$ & $\mathrm{aCD}$ & $\mathrm{abBC}$ & $\mathrm{abB}$ & $\mathrm{aA}$ \\
\hline $8 \mathrm{~L} / \mathrm{h}$ & 0.0078 & 0.01 & 0.015 & 0.019 & 0.027 & 0.044 & 0.058 & 0.037 & 0.041 & 0.046 & 0.51 & 0.61 & 0.63 & 0.72 & 0.86 \\
\hline 1 got/pl & $\mathrm{aA}$ & $\mathrm{aB}$ & $\mathrm{aC}$ & $\mathrm{aCD}$ & $\mathrm{aDE}$ & $\mathrm{aDE}$ & $\mathrm{aE}$ & $\mathrm{aB}$ & $\mathrm{abAB}$ & abA & $\mathrm{abA}$ & $\mathrm{aCD}$ & $\mathrm{abBC}$ & $\mathrm{abB}$ & $\mathrm{aA}$ \\
\hline $4 \mathrm{~L} / \mathrm{h}$ & 0.008 & 0.01 & 0.015 & 0.019 & 0.027 & 0.044 & 0.059 & 0.035 & 0.040 & 0.044 & 0.54 & 0.62 & 0.64 & 0.72 & 0.86 \\
\hline $3 \operatorname{got} / \mathrm{pl}$ & $\mathrm{aA}$ & $\mathrm{aB}$ & $\mathrm{aC}$ & $\mathrm{aD}$ & $\mathrm{aDE}$ & $\mathrm{aDE}$ & $\mathrm{aDE}$ & $\mathrm{abB}$ & $\mathrm{abAB}$ & $\mathrm{abA}$ & $\mathrm{aC}$ & $\mathrm{aBC}$ & $\mathrm{abBC}$ & $\mathrm{abB}$ & $\mathrm{aA}$ \\
\hline $4 \mathrm{~L} / \mathrm{h}$ & 0.0076 & 0.009 & 0.015 & 0.018 & 0.025 & 0.044 & 0.057 & 0.035 & 0.039 & 0.045 & 0.51 & 0.59 & 0.63 & 0.74 & 0.87 \\
\hline $2 \mathrm{got} / \mathrm{pl}$ & $\mathrm{aA}$ & $\mathrm{aB}$ & $\mathrm{aC}$ & $\mathrm{aD}$ & $\mathrm{aD}$ & $\mathrm{aE}$ & $\mathrm{aE}$ & $\mathrm{abB}$ & $\mathrm{abAB}$ & $\mathrm{abA}$ & $\mathrm{aD}$ & $\mathrm{aCD}$ & $\mathrm{abC}$ & $\mathrm{aB}$ & $\mathrm{aA}$ \\
\hline $4 \mathrm{~L} / \mathrm{h}$ & 0.0072 & 0.009 & 0.015 & 0.018 & 0.025 & 0.044 & 0.059 & 0.036 & 0.041 & 0.046 & 0.48 & 0.58 & 0.63 & 0.74 & 0.87 \\
\hline 1got/p & $\mathrm{aA}$ & $\mathrm{aB}$ & $\mathrm{aC}$ & $\mathrm{aD}$ & $\mathrm{aD}$ & $\mathrm{aE}$ & $\mathrm{aE}$ & $\mathrm{abB}$ & $\mathrm{abAB}$ & $\mathrm{abA}$ & $\mathrm{abD}$ & $\mathrm{aCD}$ & $\mathrm{abC}$ & $\mathrm{aB}$ & $\mathrm{aA}$ \\
\hline $2 \mathrm{~L} / \mathrm{h}$ & 0.0076 & 0.01 & 0.016 & 0.019 & 0.026 & 0.043 & 0.057 & 0.034 & 0.040 & 0.045 & 0.52 & 0.59 & 0.69 & 0.77 & 0.87 \\
\hline $3 \mathrm{got} / \mathrm{p}$ & $\mathrm{aA}$ & $\mathrm{aB}$ & $\mathrm{aC}$ & & $\mathrm{aD}$ & $\mathrm{aE}$ & & $\mathrm{abB}$ & & $\mathrm{abA}$ & $\mathrm{aD}$ & $\mathrm{aCD}$ & $\mathrm{aBC}$ & $\mathrm{aAB}$ & $\mathrm{aA}$ \\
\hline $2 \mathrm{~L} / \mathrm{h}$ & 0.0083 & 0.011 & 0.016 & 0.019 & 0.027 & 0.046 & 0.061 & 0.038 & 0.042 & 0.048 & 0.50 & 0.59 & 0.67 & 0.77 & 0.86 \\
\hline $2 \mathrm{got} / \mathrm{pl}$ & $\mathrm{aA}$ & $\mathrm{aB}$ & $\mathrm{aC}$ & $\mathrm{aD}$ & $\mathrm{aD}$ & $\mathrm{aE}$ & & $\mathrm{aB}$ & & $\mathrm{aA}$ & $\mathrm{aD}$ & $\mathrm{aCD}$ & $\mathrm{aBC}$ & $\mathrm{aAB}$ & $\mathrm{aA}$ \\
\hline $2 \mathrm{~L} / \mathrm{h}$ & 0.0085 & 0.01 & 0.016 & 0.019 & 0.027 & 0.046 & 0.060 & 0.036 & 0.040 & 0.046 & 0.54 & 0.62 & 0.66 & 0.77 & 0.87 \\
\hline $1 \mathrm{got} / \mathrm{pl}$ & $\mathrm{aA}$ & $\mathrm{aB}$ & $\mathrm{aC}$ & $\mathrm{aD}$ & $\mathrm{aE}$ & $\mathrm{aF}$ & $\mathrm{aG}$ & $\mathrm{abB}$ & $\mathrm{abAB}$ & $\mathrm{abA}$ & $\mathrm{aC}$ & $\mathrm{aBC}$ & $\mathrm{aBC}$ & $\mathrm{aA}$ & $\mathrm{aA}$ \\
\hline Sem & 0.0086 & 0.01 & 0.01 & 0.014 & 0.019 & 0.036 & 0.051 & 0.026 & 0.031 & 0.037 & 0.40 & 0.45 & 0.54 & 0.64 & 0.71 \\
\hline Irrig. & $\mathrm{aA}$ & $\mathrm{aB}$ & $\mathrm{bC}$ & $\mathrm{bD}$ & bDE & $\mathrm{bDE}$ & $\mathrm{bE}$ & $\mathrm{bB}$ & $\mathrm{bAB}$ & $\mathrm{bA}$ & $\mathrm{bD}$ & $\mathrm{bCD}$ & $\mathrm{bBC}$ & $\mathrm{bAB}$ & $\mathrm{bA}$ \\
\hline
\end{tabular}

* Means followed by the same lowercase letter in the column and uppercase letter in the row do not differ statistically by Tukey's test at $5 \%$ error probability level.

of the following year (March 2013), in plants with one year of planting.

Topdressing effect (Table 3) was verified on biometric variable growth rates by multiple regression, and no statistical significance was observed.

\section{DISCUSSION}

Young Brazilian mahogany (Swietenia macrophylla) plants tolerate periods of 15 to 30 days of water deficiency (Cordeiro et al., 2009). This species also belongs to the Meliaceae family, as well as the 
Table 3 - Regression analysis summary with the mean square for plant height, stem height and Diameter at breast height (DBH) of African mahogany in response to topdressing ( $\mathrm{N}$ and $\mathrm{K}$ ).

Tabela 3 - Resumo da análise de regressão com o quadrado médio, para altura de planta, altura de fuste e diâmetro à altura do peito de mogno africano, em resposta a adubação de cobertura ( $N$ e K).

\begin{tabular}{|c|c|c|c|c|}
\hline & & Plant height & Stem height & $\mathrm{DBH}$ \\
\hline Source of Variation & Degrees offreedom & Mean Square & Mean Square & Mean Square \\
\hline Linear regretion & 1 & $86.83^{\text {ns }}$ & $10.87^{\mathrm{ns}}$ & $0.005^{\mathrm{ns}}$ \\
\hline Quadratic regression & 1 & $86.80^{\mathrm{ns}}$ & $7.34^{\mathrm{ns}}$ & $0.0006^{\mathrm{ns}}$ \\
\hline
\end{tabular}

African Mahogany, although the first one is native to the Amazon biome.

Eucalyptus grandis and Pinus elliotti plants cultivated in the Triangulo Mineiro Cerrado had a higher growth when submitted to irrigation, corroborating with the results found in this study (Fernandes, 2009).

Although plants survived (95\% survival) to the water deficit period in the experimental area (Figure 1A), typical of the cerrado biome (dry season $=6$ months), this was reflected in non-irrigated plants growth, since some woody species under water deficit conditions limit stomatal opening, resulting in $\mathrm{CO}_{2}$ assimilation

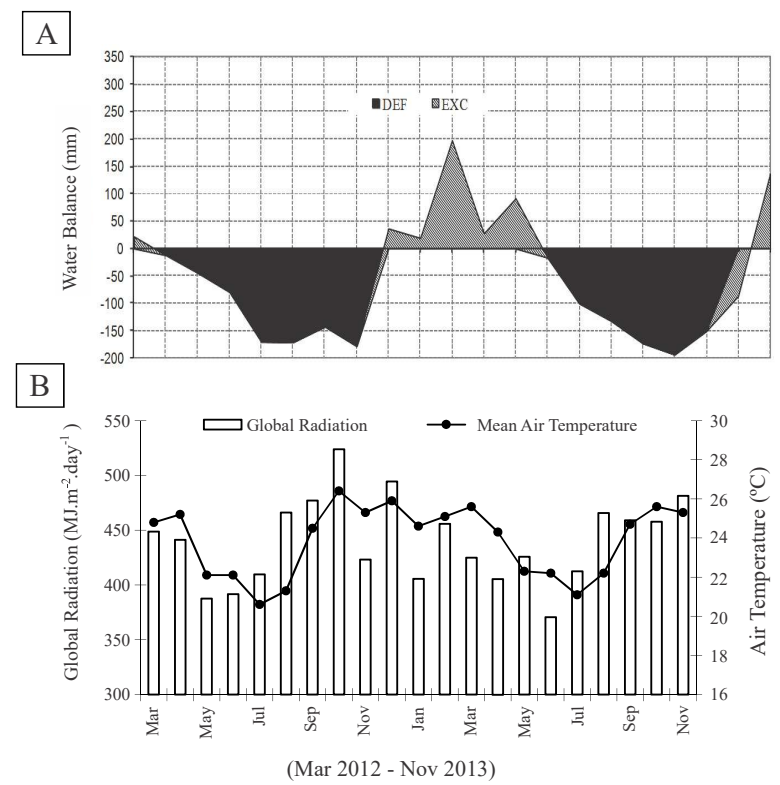

Figure 1 - A: Water balance by Thornthwaite \& Matter method (Pereira et al., 2002); and B: Temperature and global radiation for the period from March/2012 to November/2013), Bonfinópolis, GO, Brazil.

Figura 1-A: Balanço hídrico pelo método de Thornthwaite \& Matter (Pereira et al., 2002), e B: Temperatura média do ar e radiação solar para o período de Março/2012 a Novembro/2013), BonfinópolisGO. rate reduction and lower plant growth (Franco, 1998; Mattos et al., 2002; Silva et al., 2005; Albuquerque et al., 2013).

Lower non-irrigated plant growth can be explained by the planting season (March/2012), which coincided with the end of rainfall in the region. Afterwards, a drought period advanced until October/2012 (Figure 1A), which may have impaired both plant adaptation and plant growth.

Considering that the mahogany is a large species, which can reach $70 \mathrm{~m}$ height and $3.5 \mathrm{~m} \mathrm{DBH}$ (Carvalho, 2007), plants evaluated in this study are young. In addition, the radius explored by young mahogany plant roots was only $0.50 \mathrm{~m}$ until 6 months of age, and 1.0 $\mathrm{m}$ until 1 year of age. Because of this, a single dripper with a flow rate of $2 \mathrm{~L} \mathrm{~h}^{-1}$ was enough to supply plant water requirements. In treatments with 2 and 3 drippers, regardless of flow rate, drippers were arranged in a way that, in the treatment with 2 drippers, the plant was in a median region between emitters. Moreover, in the treatment with 3 drippers, the water provided by border emitters was outside the young mahogany root system water absorption zone. The distance between emitters was $60 \mathrm{~cm}$, and based on the treatment with $8 \mathrm{~L} \mathrm{~h}^{-1}$ and 3 drippers, operating 1 hour per day, a wet radius of $0.38 \mathrm{~cm}$ was obtained. That is, all the water that was expelled by border emitters was probably lost in that crop stage.

Although plant growth increased during the first months, plant growth rates intensified in the summer (December - March), probably as an influence of maximum water availability (Figure 1A) in the soil and photoperiod effect, with higher energy availability (Figure 1B).

In general, irrigated and non-irrigated treatments showed lower growth rates up to 160 days after planting (Figure 2), which may have occurred due to seedlings ripening stage and low energy availability. This effect was more accentuated in the non-irrigated treatment, since there was water restriction (Figure 1A). From 

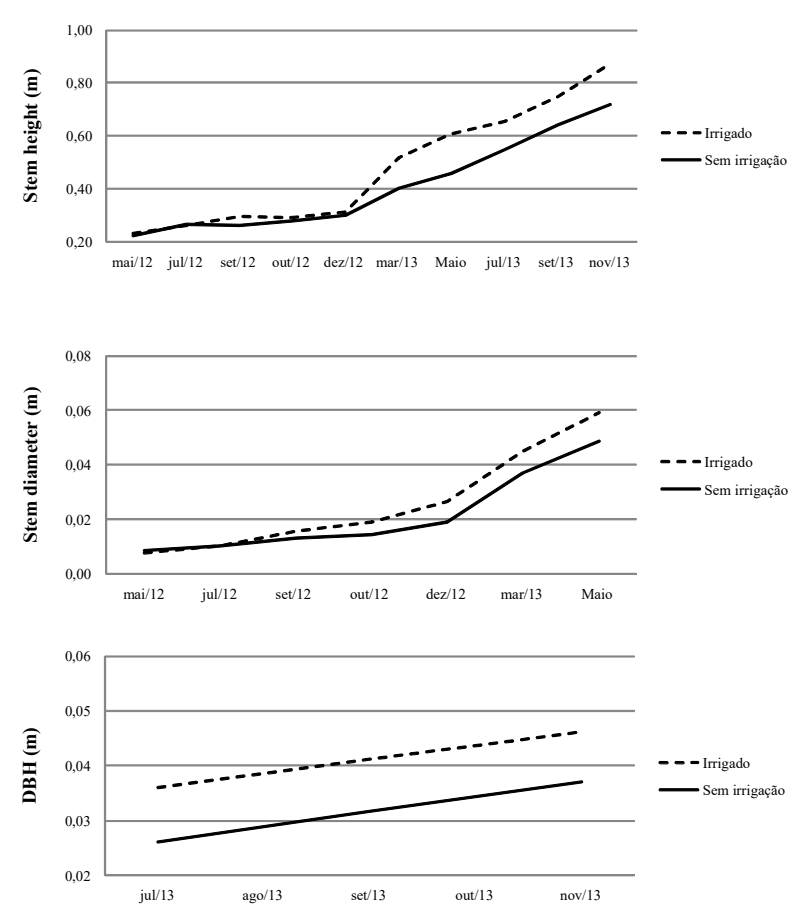

Figure 2 - Mean growth data of African mahogany trees (total height, stem height, diameter and DBH) from 2 to 20 months in the field, Bonfinópolis, GO, Brazil.

Figura 2 - Dados médios de crescimento de plantas de mogno africano (altura total, altura de fuste, diâmetro e DAP) dos 2 aos 20 meses no campo, Bonfinópolis - Go.

November/2012, higher growth rates were observed in both irrigated and non-irrigated treatments $(p<0.05)$ for all variables analyzed, decreasing again in the dry period of the following year (2013), with effect in irrigated treatments (Figure 2). Thus, the importance of irrigation for African mahogany plant growth in the first two years of forest implantation was noticed.

It was observed that, in the first reading, conducted in May 2012 (2 months of age), plants obtained mean values of $0.008 \mathrm{~m}$ diameter, $0.32 \mathrm{~m}$ total height and $0.23 \mathrm{~m}$ stem height. As of November 2013 (20 months of age), in the last evaluation, $\mathrm{DBH}$, total height and stem height mean data were, respectively: $0.045 ; 3.25$ and $0.87 \mathrm{~m}$ for irrigated plants and $0.037,2.67$ and 0.71 $\mathrm{m}$ for non-irrigated plants. In general, it was observed that mahogany plants developed slightly less than expected, including irrigated ones. The literature shows that Brazilian Mahogany plants transplanted with 0.60 $\mathrm{m}$ height, that is, approximately the double of the initial height of plants in this study $(0.32 \mathrm{~m})$, obtained a mean total height of $3.40 \mathrm{~m}$ during a period of 14 months (Cordeiro, 2012). The value aforementioned corresponds to a growth rate of $0.24 \mathrm{~m} \mathrm{month}{ }^{1}$, which was higher than the rate obtained by plants of the present study, $0.15 \mathrm{~m}$ month ${ }^{1}$ when irrigated and $0.12 \mathrm{~m} \mathrm{month}^{1}$ when not irrigated. This may be due to transplanted seedling size, or due to species and region differences. The mahogany (Swietenia macrophylla) is known as the Brazilian mahogany and is a species native to the Amazon region, where the study was conducted. Therefore, the plant is already adapted to the environment conditions.

Differences were not detected for variables that expressed plant growth in relation to the different fertilization rates (Table 3 ). In studies with Brazilian mahogany and its nutritional needs, it was verified that the order of necessity follows $\mathrm{P}>\mathrm{S}>\mathrm{K}>\mathrm{N}$ (Souza et al., 2010), which may explain the absence of a significant African mahogany response to potassium $(\mathrm{K})$ and nitrogen $(\mathrm{N})$, as these elements are not the most necessary in the initial growth of this plant. Increasing $\mathrm{N}$ doses of $0,20,40,60,80,100$ and 120 $\mathrm{g} \mathrm{N}$ ton $^{-1}$ of substrate increased Brazilian Mahogany (Swietenia macrophylla King) seedlings growth in terms of stem and root dry matter and plant height, up to the optimal dose of $61.5 \mathrm{~g} \mathrm{~N} \mathrm{Mg}^{-1}$. The maximum dose used in this study $\left(120 \mathrm{~g} \mathrm{~N} \mathrm{Mg}^{-1}\right)$ caused negative effect, impairing seedling quality (Tucci et al., 2009). Rosa (2014), in a study on African mahogany (Khaya ivorensis) seedlings in pots (90 days), reported that nitrogen fertilization only improved plant leaf area, while the other variables (height, stem and diameter) did not show significant differences between treatments $(0,00,0,12,0,24,0,36,0,48,0,60$ grams of $\mathrm{N}$ per plant $)$. Other researches with perennial species showed that $\mathrm{N}$ omission did not affect locust (Duboc et al., 1994) and aroeira-do-sertão (Myracrodruon urundeuva Fr, All) growth (Mendonça et al., 1999).

Moreover, considering that Cerrado soils are naturally low in phosphorus (Souza et al., 2004), which was confirmed in this study (4.2 and $1.4 \mathrm{mg} \mathrm{dm}^{3}$ for 0-20cm and 0-40 layers, respectively) (Fig. 1), the lack of $\mathrm{P}$ application may have caused lower growth, as it is known that phosphorus is a plant growth limiting factor for many plants, such as Cedrella fissilis (Silva and Muniz, 1995), Acacia mangium, Tibouchina 
granulosa and Aspidosperma polyneurom (Braga et al., 1995).

In addition, African mahogany can adapt to poor soils. In Indonesia, mahogany of Swietenia macrophylla King. species is developed in very poor soils (Soemianegara and Lemmens, 1993). For Carvalho (2007), there are several tolerable soil conditions for mahogany (Swietenia macrophylla), ranging from deep and poorly drained soils, acidic and swampy clay soils, to welldrained alkaline soils, including soils derived from igneous and metamorphic rocks. Due to Swietenia macrophylla and Khaya ivorensis species proximity, it can be inferred that the African mahogany is adapted to Cerrado environmental conditions. According to Sallenave (1959), who studied mahogany special characteristics and properties, this species originated in African west coast countries, mainly in Ivory Coast, Ghana, Togo, Nigeria, Cameroon, Congo and Angola. In general, the predominant soils found in these countries are classified as Oxisols, Ultisols and Entisols (Soll Survey Staff, 2006), which, according to the Brazilian classification (Embrapa, 2006), represent, respectively: Latosols, Argisols and Neosols, which are in the Cerrado of Goiás.

\section{CONCLUSIONS}

Young African mahogany plants, up to two years of age, respond positively to drip irrigation.

Irrigation with one drip per plant and flow rate of $2 \mathrm{~L} \mathrm{~h}^{-1}$ is enough to meet the water demands of African mahogany in the first two years of cultivation.

Plants did not respond to the different Nitrogen and Phosphorus topdressing doses, applied every 2 months, at the beginning of the cycle.

\section{REFERENCES}

Albuquerque MPF, Moraes FKC, Santos RIN, Castro GLS, Ramos RMLS, Pinheiro HA. Ecofisiologia de plantas jovens de mogno africano submetidas a déficit hídrico e reidratação. Pesquisa Agropecuária Brasileira. 2013;48(1):9-16.

Alves Junior J, Folegatti MV, Silva CR, Silva TJA, Evangelista AWP. Response of young 'Tahiti' lime trees to different irrigation levels. Engenharia Agrícola. 2011;31(2):303-14.

Revista Árvore. 2017;41(1):e410112
Alves Junior J, Taveira MR, Evangelista AWP, Casaroli D, Barbosa LHA. Crescimento de plantas jovens de pequizeiro irrigadas na região do Cerrado. Revista Agrotecnologia. 2013;4(1):58-73.

Braga F, Vale FR, Ventorim N, Aubert E, Lopes GA. Exigências nutricionais de quatro espécies. Revista Árvore. 1995;19(1):18-31.

Brito BV, Casaroli D, Pereira GWM, Rosa FO, Alves Junior J. Aptidão edafoclimática da cultura do Mogno Africano para o Estado de Goiás utilizando uma ferramenta SIG. In: Anais do $16^{\circ}$. Simpósio Brasileiro de Sensoriamento Remoto [cdrom]. Foz do Iguaçu: SBSR; 2013.

Carvalho PER. Mogno - Swietenia macrophylla. Colombo: Embrapa Florestas; 2007. 12p. (Circular técnico).

Cordeiro YEM. Potencial de uso em recuperação de áreas degradadas: um estudo de três espécies nativas da Amazônia Oriental sob dois regimes hídricos. Belém: 2012.89p.

Cordeiro YEM, Pinheiro HA, Santos Filho BG, Corrêa SS, Dias Filho MB. Physiological and morphological responses of young mahogany (Swietenia macrophylla King) plants to drought. Forest Ecology and Management. 2009; 258(7):1449-55.

Duboc E. Requerimentos nutricionais de espécies nativas: Hymenae acourbaril L, var, stilbocarpa (Hayne) Lee et. Lang (Jatoba) Copaiferalangsdorffiidesf, (Oleo copaíba) Peltophorus dubium (Spreng,) Taub, (Canasfistula) [dissertação]. Lavras: Universidade Federal de Lavras; 1994. 68p.

Empresa Brasileira de Pesquisa Agropecuária Embrapa. Sistema brasileiro de classificação de solos. $2^{\mathrm{a}}$.ed. Rio de Janeiro: 2006. 360p.

Fernandes ALT, Florêncio TM, De Faria MF. Recuperação de áreas degradadas Serviços Ambientais e Sustentabilidade [cd-rom]. In: Anais do $2^{\circ}$ Seminário de Recursos Hídricos da Bacia Hidrográfica do Paraíba do Sul. Taubaté: SERHIDRO-SP; 2009.

Fernandes LA, Furtini Neto AE, Fonseca FC, Vale FR. Crescimento inicial, níveis críticos de fósforo e 
frações fosfatadas em espécies florestais. Pesquisa Agropecuária Brasileira. 2000;35(6):1191-8.

Franco AC. Seasonal patterns of gas exchange, water relations and growth of Roupala montana, an ever green savana species. Plant Ecology. 1998;136(1):69-76.

Lopes JLW, Guerrini IA, Saad JCC. Qualidade de mudas de eucalipto produzidas sob diferentes lâminas de irrigação e dois tipos de substrato. Revista Árvore. 2007;31(5):835-43.

Mantovani EC, Bernardo S, Paulareti LF. Irrigação princípios e métodos. $3^{\mathrm{a}}$.ed. Viçosa, MG: Universidade Federal de Viçosa; 2009. 355p.

Mattos EA, Lobo PC, Joly CA. Overnight rainfall inducing rapid changes in photosynthetic behaviour in a "Cerrado" woody species during a dry spell amidst the rainy season. Australian Journal of Botany. 2002;50(1):241-6.

Mendonça AVR, Nogueira FD, Venturin N, Souza, JS. Exigências nutricionais de

Myracrodruonurundeuva fr, All (aroeira do sertão). Cerne. 1999;5(2):65-75.

Nogueira CCP, Coelho EF, Leão MCS. Características e dimensões do volume de um solo molhado sob gotejamento superficial e subsuperficial. Revista Brasileira de Engenharia Agrícola e Ambiental. 2000;4(3):315-20.

Pereira AR, Angelocci LR, Sentelhas PC. Agrometeorologia: fundamentos e aplicações práticas. Guaíba: Agropecuária; 2002. 487p.

Rosa FO. Mogno Africano em condições edafoclimáticas do Cerrado [dissertação]. Goiânia: Universidade Federal de Goiás; 2014. $63 \mathrm{p}$.

Sallenave NP. Caractèristique set propriétés de sacajous, revue bois et forêtsdes tropiques. Revue Bois et Forêts des Tropiques. 1959; 1(65):37-42.

Schwartzman M, Zur B. Emitter spacing and geometry of wetted soil volume. Journal of
Irrigation and Drainage Engineering. 1986;112(3):242-53.

Silva CR, Folegatti MV, Silva TJA, Alves Junior J, Souza CF, Ribeiro RV. Water relations and photosynthesis as criteria for adequate irrigation management in 'Tahiti' lime trees. Scientia Agrícola. 2005;62(5):415-22.

Silva MAG, Muniz AS. Exigências nutricionais de cedro (Cedrelafissilis velloso) em solução nutritiva. Revista Árvore. 1995;19(3):415-25.

Silva RTL. Produtividade e qualidade de frutos de goiabeira (Psidium guajava L.) irrigada por gotejamento [dissertação]. Santa Maria: Universidade Federal de Santa Maria; 2012. 100p.

Silva SC, Soares EGS, Ribeiro JR. Informações meteorológicas para pesquisa e planejamento agrícola, referentes ao município de Santo Antonio de Goiás, GO, 2006. Santo Antônio de Goiás: Embrapa Arroz e Feijão; 2007. 31p.

Soerianegara I, Lemmens RHMJ. Timber tree: major commercial timber. Plant Resources of South-East Asia. 1993;1(5):610.

Soil Survey Staff. Soil Taxonomy: a basic system of soil classification for making and interpreting soil surveys. 2nd. ed. Washington DC: Handbook 436, United States Government Printing Office; 2006. 696p. (Handbook, 436).

Sousa DMG, Lobato E, Rein TA. Adubação com fósforo. In: Sousa DMG, Lobato E. editores. Cerrado: Correção do solo e adubação. $2^{a}$.ed. Brasília: Embrapa Informação Tecnológica; 2004. p.147-68.

Souza CAS, Tucci CAF, Silva JF, Ribeiro WO. Exigências nutricionais e crescimento de plantas de mogno (Swietenia macrophylla King.). Acta Amazonica. 2010;40(3):515-22.

Tucci CAF, Lima HM, Lessa JF. Adubação nitrogenada na produção de mudas de mogno (Swietenia macrophylla King). Acta Amazonica. 2009;39(2):289-94. 
